\title{
Recent Studies on Nutrition and Parkinson's Disease Prevention: A Systematic Review
}

\author{
Ann Gaba \\ Hunter College, City University of New York School of Public Health, New York, USA \\ Email: agaba@hunter.cuny.edu
}

Received 12 March 2015; accepted 9 May 2015; published 13 May 2015

Copyright (c) 2015 by author and Scientific Research Publishing Inc.

This work is licensed under the Creative Commons Attribution International License (CC BY).

http://creativecommons.org/licenses/by/4.0/

(c) (i) Open Access

\begin{abstract}
Introduction: Parkinson's disease (PD) is a chronic progressive movement disorder, affecting primarily people over sixty years of age. With aging populations, some estimates indicate as many as nine million people will be impacted by this disease by 2030 . Thus it is imperative to identify means of prevention, delay of onset, and management of symptoms. Many elements of diet and nutrition have been investigated to accomplish these goals. This review will examine recent studies of potential diet and nutrition factors and PD incidence. Methods: A PubMed Search was conducted using the keywords "Parkinson, prevention and nutrition", and "Parkinson, prevention, and diet." Search parameters were limited to articles published in English, and published between 2005 and February 2015. Results: Nineteen studies were selected for closer evaluation, with twelve ultimately included in the analysis. Selected papers all included large samples $(\mathrm{N} \geq 200)$, comparisons of individuals with and without diagnosed PD and detailed descriptions of how nutritional intakes were assessed. Conclusions: Several specific dietary components emerged as being of potential importance in PD prevention. Dairy products, most specifically liquid whole milk, were identified as increasing PD risk. Coffee and black tea, but not green tea, seemed to be protective, most likely due to their caffeine content. Vegetables from the solenaceae family, specifically peppers, had a protective effect, possibly due to their nicotine content. Mediterranean diet adherence, with a relatively high intake of vegetables, may also be protective. The role of dietary fats is not yet clear. Dietary cholesterol was found to be protective in men, but not women, with dietary mono-unsaturated fatty acids being protective in women but not men. These results are consistent with a preliminary report for the 2015 US Dietary Guidelines, which addresses diet and risk of neurodegenerative disorders for the first time.
\end{abstract}

\section{Keywords}

Parkinson Disease, Diet, Nutrition, Prevention 


\section{Introduction}

Parkinson's disease (PD) is the second most prevalent neurodegenerative disorder, after Alzheimer's. Symptoms of PD include tremor, slowed movements, and rigidity, as well as non-motor symptoms which may include depression, changes in taste and smell perception, gastro-intestinal dysfunction, and sleep disturbance. PD is the most common movement disorder in the world, affecting about $1 \%$ of adults older than 60 years. Young-onset Parkinson's disease affects $5 \%-10 \%$ of patients [1]. PD is chronic and progressive with no known cure. Estimates of incidence vary, usually based on diagnostic criteria and sampling methods used, but it is generally accepted that this is greatly increased after age 60 [2]. With aging populations in Europe and the United States, incidence is likely to increase in coming decades. Dorsey et al. [3] estimated that the incidence of PD would increase from about 4.1 to 4.6 million worldwide in 2005, to between 8.7 and 9.3 million by 2030. Furthermore, statistics from the Parkinson's Disease Foundation [4] indicate that "the combined direct and indirect cost of Parkinson's, including treatment, Social Security payment and lost incomes from inability to work, is estimated to be $\$ 25$ billion per year in the United States alone”. Given the human and financial toll of Parkinson's disease, with increases to both anticipated, every means of decreasing incidence and morbidity should be thoroughly explored. Diet and nutrition is one such emerging area.

The Scientific Report of the 2015 Dietary Guidelines Advisory Committee [5] made mention of the potential role of diet in the etiology of neurodegenerative diseases for the first time in their history, saying: "Although the number of studies available on dietary patterns and neurodegenerative disease risk is expanding, this body of evidence, which is made up of high-quality observational studies, has appeared only in recent years and is rapidly developing”. This review examines some of this literature looking specifically at Parkinson's disease in an effort to identify significant diet and nutrition factors that may be related to prevention or delayed onset of PD.

\section{Methods}

\subsection{Search Methodology}

A Pub Med Search was conducted using the keywords "Parkinson, prevention and nutrition," and "Parkinson, prevention, and diet." Search parameters were limited to articles published in English, and published between 2005 and February 2015 (See Figure 1).

\subsection{Study Selection}

The key words "Parkinson, prevention and nutrition" yielded 55 results, with "Parkinson, prevention and diet" producing 82 results. Further searches using similar terms did not yield any additional relevant documents. From these two initial lists of articles, after duplicate citations were removed, nineteen articles were selected for closer evaluation and of these, twelve articles met all inclusion criteria and were included for analysis. The twelve selected papers all included large samples $(\mathrm{N}>200)$, with comparisons of individuals with and without diagnosed PD. These studies also gave detailed descriptions of how nutritional intakes were assessed (Table 1).

\subsection{Data Extraction}

Data was extracted from the selected published papers. Authors were not contacted. Data obtained included: diet/nutritional factor(s) under study, population, sample size, diet intake/assessment methodology, primary findings including hazard ratio (HR), odds ratio (OR) and/or relative risk (RR) statistics as available and the accompanying $95 \%$ confidence intervals for these.

\section{Results}

Four of these studies, one by Chen et al. [6] and three by Palacias et al. [7]-[9] used data from the American Cancer Society's Cancer Prevention Study II Nutrition Cohort. This cohort was established by the American Cancer Society in 1992 for a prospective study investigating risk factors for cancer incidence [10]. The total population included 86,404 men and 97,786 women. Of these, 250 men and 138 women with Parkinson's disease were identified during follow-up (1992-2001).

The Nutrition Cohort described above was a subgroup of the 1.2 million US men and women originally recruited in 1982 for research on cancer mortality in Cancer Prevention Study II. Participants were aged 50 to 74 


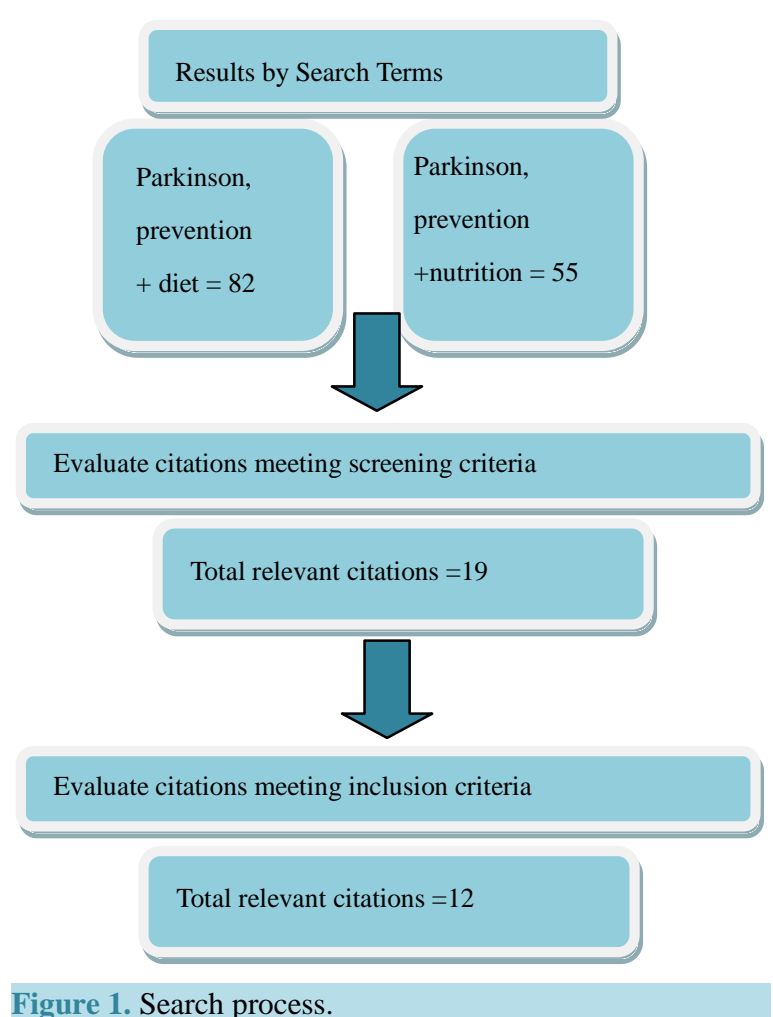

years at enrollment in 1992 or 1993, when they completed a mailed questionnaire that included questions on demographic, medical, environmental, and lifestyle factors and dietary habits. Follow-up surveys were conducted in 1997, 1999, and 2001 to ascertain whether any major chronic diseases had been diagnosed. Diet was assessed at baseline using a 68-item semiquantitative food frequency questionnaire that was modified from the brief Health Habits and History Questionnaire developed by Block et al. [11]. This food frequency questionnaire was also validated in a subgroup of 441 participants against four random 24-hour dietary recalls completed over a 1-year period [12].

Analysis of data from this population by Chen et al. [6] found that dairy product consumption was positively associated with risk of Parkinson's disease: Compared with the lowest intake quintile, the corresponding relative risks (RR) for quintiles 2 to 5 were 1.4, 1.4, 1.4, and 1.6 (95 percent confidence interval (CI): 1.1, 2.2; p for trend $=0.05)$.

For this same cohort, Palacios and colleagues examined three possible relationships to PD. Their first analysis [7] found that neither body mass index nor waist circumference significantly predicted Parkinson's disease risk. Relative risk comparing individuals with a baseline body mass index of $\geq 30$ to those with a body mass index $<23$ was 1.00 (95\% confidence interval: $0.75,1.34$; p trend $=0.79$ ), and that comparing individuals with a waist circumference in the top category ( $\geq 40.3$ inches in men and $\geq 35$ inches in women) to those in the bottom category (<34.5 inches in men and $<28$ inches in women) was 1.35 (95\% confidence interval: $0.95,1.93 ; \mathrm{p}$ trend = 0.08).

History of diabetes was also not significantly associated with Parkinson's disease risk (combined relative risks $=0.88$; 95\% confidence interval: 0.62, 1.25; p heterogeneity $=0.96$ ). In addition, neither body mass index at age 18 nor changes in weight between age 18 and baseline were significantly associated with Parkinson’s disease risk. The results did not differ significantly by gender.

They [8] also analyzed data from this cohort to examine alcohol intake and risk of PD. After adjustment for age, smoking, and other risk factors, the RR comparing men consuming 30 or more grams of alcohol per day (highest category) to nondrinker men was 1.29 (95\% confidence interval [CI]: 0.90, 1.86; p trend = 0.40), and the RR comparing women consuming 15 or more grams of alcohol (highest category) per day to nondrinker women was 0.77 (95\% CI: 0.41, 1.45; p trend = 0.87). Consumption of specific forms of alcohol, beer, wine, or liquor, was also not associated with PD risk. 


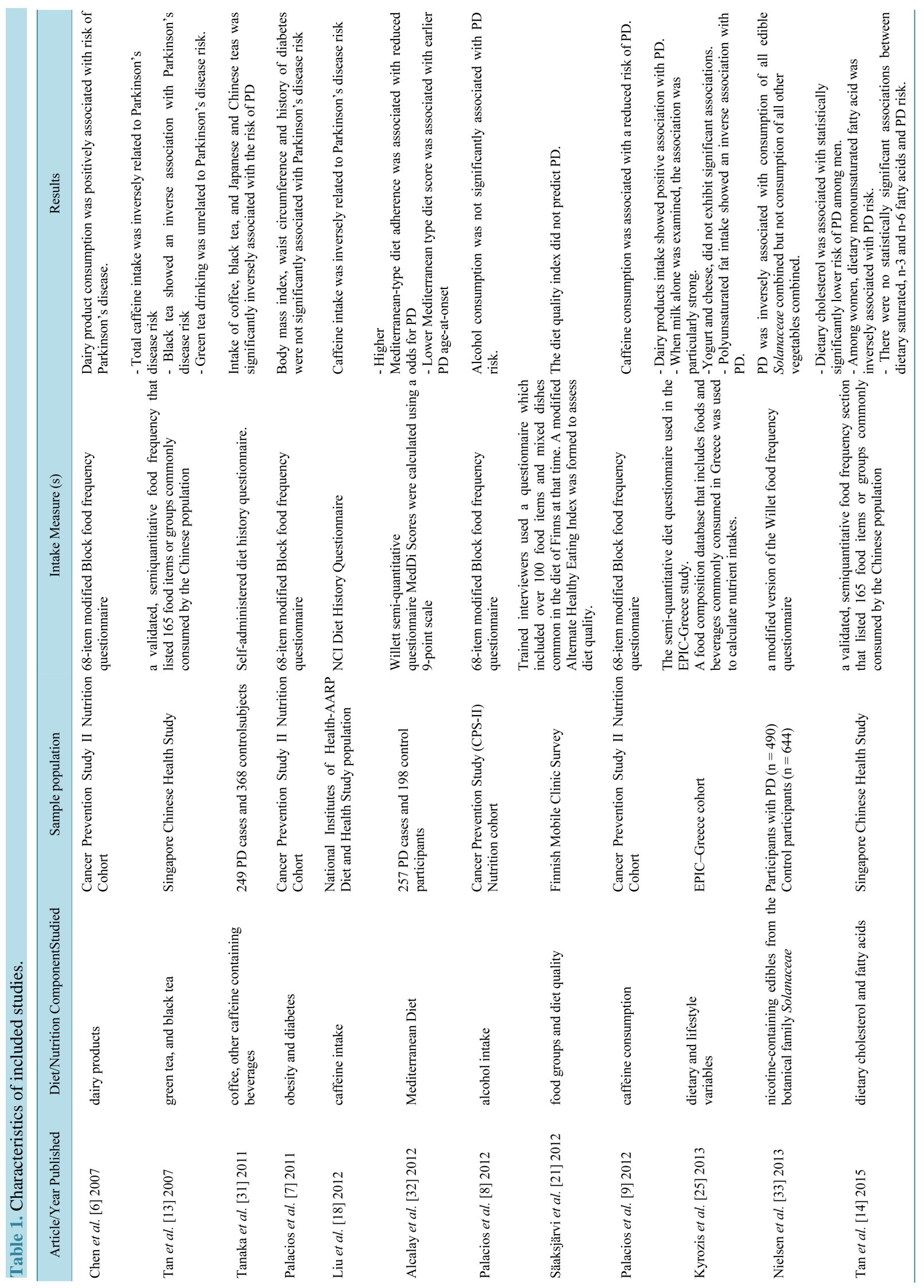


In their third study [9] they examined the relationship between caffeine intake and PD. They found that after adjustment for age, smoking, and alcohol intake, high caffeine consumption was associated with a reduced risk of PD. The RR comparing the 5th to the 1st quintile of caffeine intake was 0.43 (95\% confidence interval [CI]: 0.26, 0.71; p trend $=0.002)$ in men, and $0.61(95 \%$ CI: 0.34, 1.09; p trend $=0.05)$ in women. Among women, this association was stronger among never users of hormone replacement therapy (HRT) $(R R=0.32)$ than among ever users $(\mathrm{RR}=0.81$; $\mathrm{p}$ interaction $=0.15)$. Consumption of decaffeinated coffee was not associated with PD risk.

Two studies by LC Tan et al. [13] and K Tan et al. [14] used data from the Singapore Chinese Health Study. This is a population based cohort that was established between April 1993 and December 1998 by recruiting 63,257 ethnic Chinese aged 45 to74 years, belonging to the two major dialect groups of Hokkien and Cantonese, and residing in government-built housing estates, where the majority of the Chinese population was living at the time [15].

For these studies, dietary intakes were evaluated using a validated, semiquantitative food frequency. This instrument listed 165 food items or groups commonly consumed by the Chinese population, with accompanying photographs and including eight food frequency categories and a choice three portion sizes. This food frequency questionnaire had been validated against a series of 24-hour dietary recall interviews [15] and selected biomarker studies [16] [17].

The study by LC Tan et al. [13] found that total caffeine intake was inversely related to Parkinson's disease risk ( $\mathrm{p}$ for trend $=0.002$ ); the relative risk for the highest versus lowest quartile was 0.55 (95\% confidence interval: 0.35, 0.88). Black tea showed an inverse association with Parkinson's disease risk that was not confounded by total caffeine intake or tobacco smoking ( $\mathrm{p}$ for trend $=0.0006$; adjusted relative risk for the highest vs. lowest tertile of intake $=0.29,95 \%$ confidence interval: $0.13,0.67$ ). Green tea drinking was unrelated to Parkinson's disease risk. For the same population, K Tan et al. [14] reported that dietary cholesterol was associated with statistically significantly lower risk of PD in a dose-dependent manner among men after adjustment for established risk factors for PD and intakes of major fatty acids. Compared to the lowest quartile, HR (95\% $\mathrm{CI}$ ) for the highest quartile was $0.53(95 \% \mathrm{CI} 0.33$ to 0.84$)$ (p for trend $=0.006)$. Among women, dietary monounsaturated fatty acid was inversely associated with PD risk ( $\mathrm{p}$ for trend $=0.033$ ). Compared to the lowest quartile, HR for the highest quartile was 0.44 (95\% CI 0.22, 0.88). They found no statistically significant association between dietary saturated fat, n-3 and n-6 fatty acids and PD risk.

Liu et al. [18] used data from the NIH-AARP Diet and Health Study which was established in 1995-1996 by the National Cancer Institute to investigate the roles of diet and lifestyle in cancer etiology [19]. This cohort was comprised of 566,401 AARP members aged 50 to 71 years from six US states and two metropolitan areas who answered a baseline survey on diet and lifestyle. A follow-up survey was conducted from 2004 to 2006 among the surviving participants in the cohort to update lifestyle exposures and to ascertain the occurrence of major chronic diseases, including PD. A total of 318,260 participants (187,499 men and 130,761 women) responded to the follow-up survey and were thus eligible for the study by Liu. A total of 2432 participants reported a PD diagnosis on the follow-up questionnaire. Data on caffeine intake (mg/day) were derived from the cohort's baseline survey. This study examined data from participants in the follow up survey. Of these, 2432 participants reported a PD diagnosis. Data on caffeine intake (mg/day) were derived from the cohort's baseline survey, which included the Diet History Questionnaire, developed by National Cancer Institute investigators [20]. They found that after adjustment for age, race, and physical activity, the odds ratio (OR) comparing the highest quintile of caffeine intake with the lowest was 0.75 (95\% confidence interval: 0.60, 0.94; p trend $=0.005$ ) for men and 0.60 (95\% confidence interval: 0.39, 0.91; p trend $=0.005$ ) for women. Further adjustment for duration of smoking and analyses carried out among never smokers showed similar results.

Säaksjärvi et al. [21] investigated diet and PD in the Finnish Mobile Clinic Survey. A total of 62,440 individuals aged 15 years or over participated (82.5\% of those invited) in the Finnish Mobile Clinic Health Examination Survey (FMC) conducted in different regions of Finland in from 1966 to 1972 [22]. As part of the main study, detailed data about food consumption was collected from 10,054 randomly selected individuals using a dietary history interview [23] [24]. Of these, participants aged 40 to 79 years were included in this cohort study on PD $(n=4738)$. All participants in the cohort were free of PD at baseline. Data collection included health examinations, a questionnaire and a one-year dietary history interview. In addition to intake of specific foods, aggregate diet quality was assessed by creating an Alternate Healthy Eating Index (AHEI). During a 41-year follow-up, eighty-five incident cases of PD were identified. Statistically significant positive associations were 
found between PD incidence for fruits and berries in men and milk in women. An inverse association between the intake of meat products and PD was found in women. The AHEI did not predict PD, the adjusted relative risk between the highest and lowest quartiles being 1.83 (95\% CI 0.65, 5.18) in men and 0.97 (95\% CI 0.38, 2.48) in women.

Kyrozis et al. [25] used the European Prospective Investigation into Cancer and Nutrition Greek cohort, (EPIC-Greece) to identify dietary and lifestyle variables associated with PD, focusing particularly on those variables identified with PD in other studies. The EPIC-Greece population-based cohort, consisted of 28,572 volunteers, 16,618 women (58.2\%) and 11,954 men (41.8\%), enrolled between 1993 and 1999 while aged between 20 and 86 years. Kyrozis and colleagues used data from EPIC follow-up surveys until June 2009.

The EPIC investigators assessed dietary variables at recruitment to evaluate intake during the year preceding enrolment. They used a semi-quantitative questionnaire including approximately 150 foods and beverages, which was administered by trained interviewers [26] [27]. Using this measure, diet during the first year had been shown to substantially approximate diet during several years preceding or following study enrolment [28] [29]. The questionnaire had also been validated during the pilot phase of the EPIC-Greece study [26] [27]. A food composition database that included foods and beverages commonly consumed in Greece was used to calculate nutrient intakes [30]. Food groups and nutrients examined in association with PD included lipids (total, saturated, monounsaturated and polyunsaturated fat), total protein, total carbohydrate, dairy products (total intake of milk, yogurt and cheese), fish \& seafood, cholesterol, vitamin D, total vegetables, total meat and total cereals.

Results of this analysis indicated that intake of dairy products was positively associated with PD. When milk alone was examined, this association was particularly strong $(\mathrm{HR}=1.34,95 \% \mathrm{CI}=1.14-1.58, \mathrm{p}=0.001)$. Separate analysis of other dairy products common in the Greek diet, yogurt and cheese, found no significant associations. Among lipids, polyunsaturated fat intake showed an inverse association with PD. (HR was not given.) Caffeinated coffee drinking was strongly inversely associated with PD in a univariate model, but not in age adjusted models. Likewise, body mass index (BMI) was not significantly associated with PD in this analysis.

In addition to these population-based epidemiologic studies, three large case-control studies were also examined [31]-[33]. Tanaka et al. [31] assessed the association of the intake of coffee, other caffeine containing beverages, and caffeine with the risk of PD in Japan, in a case-control study including 249 PD cases and 368 control subjects. Dietary habits during the month preceding enrollment were assessed using a self-administered, semi-quantitative, diet history questionnaire (DHQ). Estimates of daily intake of foods (150 items in total), energy, and selected nutrients were calculated using an ad hoc computer algorithm for the DHQ based on the Standard Tables of Food Composition in Japan [34] [35]. Adjustments to the models were made for sex, age, region of residence, educational level, pack-years of smoking, body mass index, the dietary glycemic index, as well as intake of cholesterol, vitamin E, b-carotene, vitamin B6, alcohol, and iron. Their results showed that intakes of coffee, black tea, and Japanese and Chinese teas were significantly inversely associated with the risk of PD: the adjusted odds ratios in comparison of the highest with the lowest quartile were $0.52,0.58$, and 0.59 , respectively (95\% confidence intervals $=0.30-0.90,0.35-0.97$, and $0.35-0.995$, respectively).

Alcalay et al. [32] investigated the possible relationship of the Mediterranean diet pattern (Med Di) and PD. They defined the Mediterranean diet as characterized by a high intake of vegetables, legumes, fruits, and cereals; a high intake of unsaturated fatty acids (mostly from olive oil); a moderately high intake of fish; a low to-moderate intake of dairy products, meat and poultry; and a regular but moderate consumption of ethanol, primarily in the form of wine and generally during meals [36] [37]. 257 PD participants and 198 controls, recruited by the authors, completed the Willett semi-quantitative food frequency questionnaire to quantify diet intake during the past year. Scores for Med Di adherence were calculated using a 9-point scale. Higher scores indicated greater adherence to the Mediterranean-type diet. Logistic regression models were used to assess the association between PD status and Mediterranean-type diet, adjusting for caloric intake, age, gender, education and ethnicity. Adjusted linear regression models were used to examine the association between Mediterranean-type diet adherence and PD age-at-onset. Higher Med Di adherence scores were associated with reduced odds for PD after adjustment for all covariates $(\mathrm{OR}=0.86,95 \% \mathrm{CI}=0.77-0.97, \mathrm{p}=0.010)$. Lower Med Di scores were associated with earlier age-at-onset for the PD subjects $(\beta=1.09, \mathrm{p}=0.010)$.

Nielsen et al. [33] compared 490 newly diagnosed idiopathic PD cases diagnosed during 1992 to 2008 with 644 unrelated, neurologically normal controls. They investigated whether PD was associated with self-reported frequency of consumption of foods from the Solanaceae family: including peppers, tomatoes, tomato juice, and potatoes. They adjusted for consumption of other vegetables, age, sex, race/ethnicity, tobacco use, and caffeine 
in their statistical model. PD was inversely associated with consumption of all edible Solanaceae combined (relative risk $(\mathrm{RR})=50.81,95 \%$ confidence interval $[\mathrm{CI}]=0.65-1.01$ per time per day), but not consumption of all other vegetables combined $(\mathrm{RR}=1.00,95 \% \mathrm{CI}=0.92-1.10)$. The trend strengthened when weighted by the Solanaceae nicotine concentration $(\mathrm{p}$ trend $=0.004)$. An inverse association was found specifically for peppers ( $\mathrm{p}$ trend $=0.005$ ). The potentially protective effect of edible Solanaceae was most evident in individuals who had little or no exposure to tobacco.

\section{Discussion}

The limitations of observational studies are well known [38] [39]. Therefore any potential relationships thus identified must be interpreted with caution. However, when multiple studies concur that a relationship exists, more detailed investigation is warranted. For example, the 2015 Dietary Guidelines Advisory Committee (DGAC) [5] reported that "studies appearing in the last few years reflect increasing research interest in the links between diet and neurological health." Furthermore, "A more substantial literature on dietary patterns and neuropsychological health has been published since 2010. The DGAC was therefore able to consider prevention of adult neuropsychological ill health for the first time.” In addition to the above, they also note that many recommendations from studies of diet and neurological health are consistent with general recommendations for healthy people, such as increased intake of fruits and vegetables. Two of the studies reviewed here [32] [33] support a potential role for increased vegetable consumption as being protective against PD.

More unique to the possible dietary links to PD are the results regarding dairy products [6] [25]. While a theory to explain these results is lacking, they are consistent with the findings of an older study [40]. Similarly, the results for caffeine intake [9] [13] [31] and nicotine intake [33] are similar to those reported by previous literature [41]-[45]. Whether this will develop into a warrant for a recommendation that people consume only black coffee, and to do so a specific number of times per day remains to be seen.

\section{Conclusion}

With the prevalence of PD projected to increase concurrently with an aging population, identifying potential protective factors is likely to be valuable. Several large studies of human populations have identified dietary components as impacting the risk of developing PD. For the most consistently occurring variables, prospective studies with robust and detailed measures of dietary intake would help to clarify dietary risk factors for PD. As evidence for a role of diet and nutrition in neurodegenerative disease mounts, it is likely to provide a valuable contribution to public health nutrition recommendations such as the DGA.

\section{Acknowledgements}

The author received no financial support for the work described in this article. The author is solely responsibility for the contents.

\section{References}

[1] Samii, A., Nutt, J.G. and Ransom, B.R. (2004) Parkinson’s Disease. Lancet, 363, 1783-1793. http://dx.doi.org/10.1016/S0140-6736(04)16305-8

[2] De Lau, L.M. and Breteler, M.M.B. (2006) Epidemiology of Parkinson’s Disease. The Lancet Neurology, 5, 525-535. http://dx.doi.org/10.1016/S1474-4422(06)70471-9

[3] Dorsey, E.R., Constantinescu, R., Thompson, J.P., Biglen, K.M., Holloway, R.G., Rieburtz, K., et al. (2007) Projected Number of People with Parkinson Disease in the Most Post Populous Nations, 2005 through 2030. Neurology, 68, 384-386. http://dx.doi.org/10.1212/01.wnl.0000247740.47667.03

[4] www.pdf.org/en/parkinson_statistics

[5] Scientific Report of the 2015 Dietary Guidelines Advisory Committee. http://www.health.gov/dietaryguidelines/2015-scientific-report/

[6] Chen, H., O’Reilly, E., McCullough, M.L., Rodriguez, C., Schwarzschild, M.A., Calle, E.E., et al. (2007) Consumption of Dairy Products and Risk of Parkinson's Disease. American Journal of Epidemiology, 165, 998-1006. http://dx.doi.org/10.1093/aje/kwk089

[7] Palacios, N., Gao, X., McCullough, M.L., Jacobs, E.J., Patel, A.V., Mayo, T., et al. (2011) Obesity, Diabetes, and Risk 
of Parkinson’s Disease. Movement Disorders, 26, 2253-2259.

[8] Palacios, N., Gao, X., O’Reilly, E., Schwarzschild, M., McCullough, M.L., Mayo, T., et al. (2012) Alcohol and Risk of Parkinson's Disease in a Large, Prospective Cohort of Men and Women. Movement Disorders, 27, 980-987.

[9] Palacios, N., Gao, X., McCullough, M.L., Schwarzschild, M.A., Shah, R., Gapstur, S. and Ascherio, A. (2012) Caffeine and Risk of Parkinson's Disease in a Large Cohort of Men and Women. Movement Disorders, 27, 1276-1282.

[10] Calle, E.E., Rodriguez, C., Jacobs, E.J., et al. (2002) The American Cancer Society Cancer Prevention Study II Nutrition Cohort: Rationale, Study Design, and Baseline Characteristics. Cancer, 94, 2490-2501. http://dx.doi.org/10.1002/cncr.101970

[11] Block, G., Hartman, A.M. and Naughton, D. (1990) A Reduced Dietary Questionnaire: Development and Validation. Epidemiology, 1, 58-64. http://dx.doi.org/10.1097/00001648-199001000-00013

[12] Flagg, E.W., Coates, R.J., Calle, E.E., et al. (2000) Validation of the American Cancer Society Cancer Prevention Study II Nutrition Survey Cohort Food Frequency Questionnaire. Epidemiology, 11, 462-468. http://dx.doi.org/10.1097/00001648-200007000-00017

[13] Tan, L.C., Koh, W.P., Yuan, J.M., Wang, R., Au, W.L., Tan, J.H., Tan, E.K. and Yu, M.C. (2007) Differential Effects of Black versus Green Tea on Risk of Parkinson's Disease in the Singapore Chinese Health Study. American Journal of Epidemiology, 167, 553-560. http://dx.doi.org/10.1093/aje/kwm338

[14] Tan, K., Methawasin, K., Tan, E.K., Tan, J.H., Au, W.L., Yuan, J.M. and Koh, W.P. (2015) Dietary Cholesterol, Fats and Risk of Parkinson's Disease in the Singapore Chinese Health Study. Journal of Neurology, Neurosurgery Psychiatry.

[15] Hankin, J.H., Stram, D.O., Arakawa, K., et al. (2001) Singapore Chinese Health Study: Development, Validation, and Calibration of the Quantitative Food Frequency Questionnaire. Nutrition and Cancer, 39, 187-195. http://dx.doi.org/10.1207/S15327914nc392_5

[16] Seow, A., Shi, C.Y., Franke, A.A., et al. (1998) Isoflavonoid Levels in Spot Urine Are Associated with Frequency of Dietary Soy Intake in a Population-Based Sample of Middle-Aged and Older Chinese in Singapore. Cancer Epidemiology, Biomarkers Prevention, 7, 135-140.

[17] Seow, A., Shi, C.Y., Chung, F.L., et al. (1998) Urinary Total Isothiocyanate (ITC) in a Population-Based Sample of Middle-Aged and Older Chinese in Singapore: Relationship with DIETARY total ITC and Glutathione S-Transferase M1/T1/P1 Genotypes. Cancer Epidemiology, Biomarkers Prevention, 7, 775-781.

[18] Liu, R., Guo, X., Park, Y., Huang, X., Sinha, R., Freedman, N.D., et al. (2012) Caffeine Intake, Smoking, and Risk of Parkinson Disease in Men and Women. American Journal of Epidemiology, 175, 1200-1207. http://dx.doi.org/10.1093/aje/kwr451

[19] Schatzkin, A., Subar, A.F., Thompson, F.E., et al. (2001) Design and Serendipity in Establishing a Large Cohort with Wide Dietary Intake Distributions: The National Institutes of Health-American Association of Retired Persons Diet and Health Study. American Journal of Epidemiology, 154, 1119-1125. http://dx.doi.org/10.1093/aje/154.12.1119

[20] Subar, A.F., Thompson, F.E., Kipnis, V., et al. (2001) Comparative Validation of the Block, Willett, and National Cancer Institute Food Frequency Questionnaires: The Eating at America’s Table Study. American Journal of Epidemiology, 154, 1089-1099. http://dx.doi.org/10.1093/aje/154.12.1089

[21] Sääksjärvi, K., Knekt, P., Lundqvist, A., Männistö, S., Heliövaara, M., Rissanen, H. and Järvinen, R. (2013) A Cohort Study on Diet and the Risk of Parkinson's Disease: The Role of Food Groups and Diet Quality. British Journal of Nutrition, 109, 329-337. http://dx.doi.org/10.1017/S0007114512000955

[22] Aromaa, A. (1981) Epidemiology and Public Health Impact of High Blood Pressure in Finland (in Finnish with an English summary). The Social Insurance Institution, Helsinki, Finland, Series AL 17.

[23] Järvinen, R., Seppänen, R. and Knekt, P (1993) Short-Term and Long-Term Reproducibility of Dietary History Interview Data. International Journal of Epidemiology, 22, 520-527. http://dx.doi.org/10.1093/ije/22.3.520

[24] Järvinen, R., Knekt, P., Seppänen, R., Reunanen, A., Heliövaara, M., Maatela, J. and Aromaa, A. (1994) Antioxidant Vitamins in the Diet: Relationships with Other Personal Characteristics in Finland. Journal of Epidemiology and Community Health, 48, 549-554.

[25] Kyrozis, A., Ghika, A., Stathopoulos, P., Vassilopoulos, D., Trichopoulos, D. and Trichopoulou, A. (2013) Dietary and Lifestyle Variables in Relation to Incidence of Parkinson's Disease in Greece. European Journal of Epidemiology, 28, 67-77. http://dx.doi.org/10.1007/s10654-012-9760-0

[26] Gnardellis, C., Trichopoulou, A., Katsouyanni, K., Polychronopoulos, E., Rimm, E.B. and Trichopoulos, D. (1995) Reproducibility and Validity of an Extensive Semiquantitative Food Frequency Questionnaire among Greek School Teachers. Epidemiology, 6, 74-77. http://dx.doi.org/10.1097/00001648-199501000-00015

[27] Katsouyanni, K., Rimm, E.B., Gnardellis, C., Trichopoulos, D., Polychronopoulos, E. and Trichopoulou, A. (1997) 
Reproducibility and Relative Validity of an Extensive Semi-Quantitative Food Frequency Questionnaire Using Dietary Records and Biochemical Markers among Greek Schoolteachers. International Journal of Epidemiology, 26, S118S127. http://dx.doi.org/10.1093/ije/26.suppl 1.S118

[28] Goldbohm, R.A., van der Veer, P., van den Brandt, P.A., et al. (1995) Reproducibility of a Food Frequency Questionnaire and Stability of Dietary Habits Determined from Five Annually Repeated Measurements. European Journal of Clinical Nutrition, 49, 420-429.

[29] Willett, W.C., Sampson, L., Browne, M.L., et al. (1988) The Use of a Self Administered Questionnaire to Assess Diet 4 Years in the Past. American Journal of Epidemiology, 127, 188-199.

[30] Trichopoulou, A. and Georga, K., Eds. (2004) Composition Tables of Simple and Composite Foods. Parisianos, Athens, Greece.

[31] Tanaka, K., Miyake, Y., Fukushima, W., Sasaki, S., Kiyohara, C., Tsuboi, Y., et al. (2011) Intake of Japanese and Chinese Teas Reduces Risk of Parkinson's Disease. Parkinsonism \& Related Disorders, 17, 446-450. http://dx.doi.org/10.1016/j.parkreldis.2011.02.016

[32] Alcalay, R.N., Gu, Y., Mejia-Santana, H., Cote, L., Marder, K.S. and Scarmeas, N. (2012) The Association between Mediterranean Diet Adherence and Parkinson's Disease. Movement Disorders, 27, 771-774. http://dx.doi.org/10.1002/mds.24918

[33] Nielsen, S.S., Franklin, G.M., Longstreth Jr., W.T., Swanson, P.D. and Checkoway, H. (2013) Nicotine from Edible Solanaceae and Risk of Parkinson Disease. Annals of Neurology, 74, 472-477. http://dx.doi.org/10.1002/ana.23884

[34] Science and Technology Agency (2005) Standard Tables of Food Composition in Japan. 5th Revised and Enlarged Edition, Printing Bureau of the Ministry of Finance, Tokyo. (In Japanese)

[35] Science and Technology Agency (2005) Standard Tables of Food Composition in Japan, Fatty Acids Section. 5th Revised and Enlarged Edition, Printing Bureau of the Ministry of Finance, Tokyo. (In Japanese)

[36] Scarmeas, N., Stern, Y., Tang, M.X., Mayeux, R. and Luchsinger, J.A. (2006) Mediterranean Diet and Risk for Alzheimer's Disease. Annals of Neurology, 59, 912-921. http://dx.doi.org/10.1002/ana.20854

[37] Trichopoulou, A., Costacou, T., Bamia, C. and Trichopoulos, D. (2003) Adherence to a Mediterranean Diet and Survival in a Greek Population. The New England Journal of Medicine, 348, 2599-2608. http://dx.doi.org/10.1056/NEJMoa025039

[38] Pocock, S.J. and Elbourne, D.R. (2000) Randomized Trials or Observational Tribulations? The New England Journal of Medicine, 342, 1907-1909. http://dx.doi.org/10.1056/NEJM200006223422511

[39] Grimes, D.A. and Schultz, K.F. (2002) Bias and Causal Associations in Observational Research. The Lancet, 359, 248-252. http://dx.doi.org/10.1016/S0140-6736(02)07451-2

[40] Chen, H., Zhang, S.M., Hermain, M.A., Willett, W. and Ascherio, A. (2002) Diet and Parkinson’s Disease: A Potential Role of Dairy Products in Men. Annals of Neurology, 52, 793-801. http://dx.doi.org/10.1002/ana.10381

[41] Hernán, M.A., Takkouche, B., Caamaño-Isorna, F. and Gestal-Otero, J.J. (2002) A Meta-Analysis of Coffee Drinking, Cigarette Smoking, and the Risk of Parkinson's Disease. Annals of Neurology, 52, 276-284. http://dx.doi.org/10.1002/ana.10277

[42] Tan, E.K., Fook-Chong, S.M.C., Lum, S.Y., Chai, A., Chung, H., Shen, H., Zhao, Y., Teoh, M.L., Yih, Y., Pavanni, R., Chandran, V.R. and Wong, M.C. (2003) Dose-Dependent Effect of Coffee, Tea, and Smoking in Parkinson's Disease: A Study in Ethnic Chinese. Journal of the Neurological Sciences, 216, 163-167. http://dx.doi.org/10.1016/j.jns.2003.07.006

[43] Ross, G.W., Abbott, R.D., Petrovich, H., Morens, D.M., Grandinetti, A., Tung, K.H., Tanner, C.M., Masaki, K.H., Blanchette, P.L., Curb, J.D., Popper, J.S. and White, L.R. (2000) Association of Coffee and Caffeine Intake with the Risk of Parkinson Disease. JAMA, 283, 2674-2679. http://dx.doi.org/10.1001/jama.283.20.2674

[44] Ascherio, A., Zhang, S.M., Hernan, M.A., Kawachi, I., Colditz, G.A., Speizer, F.E. and Willett, W.C. (2001) Prospective Study of Caffeine Consumption and Risk of Parkinson's Disease in Men and Women. Annals of Neurology, 50, 56-63. http://dx.doi.org/10.1002/ana.1052

[45] Ascherio, A., Weisskopf, M.G., O’Reilly, E.J., McCullough, M.L., Calle, E.E., Rodriguez, C. and Thun, M.J. (2004) Coffee Consumption, Gender, and Parkinson's Disease Mortality in the Cancer Prevention Study II Cohort: The Modifying Effects of Estrogen. American Journal of Epidemiology, 160, 977-984. http://dx.doi.org/10.1093/aje/kwh312 